\title{
Langues et démocratie : un lien imprescriptible
}

\section{Gilbert Dalgalian}

\section{(2) OpenEdition}

\section{Journals}

Édition électronique

URL : http://journals.openedition.org/esp/1654

DOI : 10.4000/esp. 1654

ISSN : 2532-0319

\section{Éditeur}

Centre d'Information sur l'Éducation Bilingue et Plurilingue

\section{Édition imprimée}

Date de publication : 1 décembre 2017

Pagination : 93-95

ISSN : 1127-266X

\section{Référence électronique}

Gilbert Dalgalian, "Lanques et démocratie : un lien imprescriptible», Éducation et sociétés plurilingues

[En ligne], 43 | 2017, mis en ligne le 01 février 2019, consulté le 15 septembre 2020. URL : http:// journals.openedition.org/esp/1654 


\section{LANGUES ET DÉMOGRATIE: UN LIEN IMPRESCRIPTIBLE}

\section{Gilbert Dalgalian}

Il legame fra lo statuto delle lingue e la democrazia non è solo di natura istituzionale; è più profondo, più fondamentale, perché si tratta di un legame morale e filosofico. La lingua è al cuore dell'individuo, al tempo stesso sua espressione personale e sua voce - e sua via di comunicazione - verso l'Altro. Partendo da ciò, la lingua ben più che il proprio statuto sociale, professionale ed economico - è per ciascuno indissociabile dal proprio essere al mondo, dalla propria personalità. Essa resta un punto di ancoraggio che si può raramente perdere, anche se durante tutta la vita si possono aggiungere punti di ancoraggio supplementari, vale a dire seconde lingue.

Parole chiave: statuto delle lingue, democrazia, radici, punto di ancoraggio

The link between the status of a language and democracy is not only an institutional issue, it is more fundamental because it is moral and philosophical. Language is the core of the individual, both his/her personal expression and means of reaching the Other. More than a symbol of a social, professional or economic status, language is inseparable from one's way of being in the world. It remains one's permanent anchor, even if one can add other anchor points to it as life unfolds - second languages.

Keyzerds: language status, Democracy, roots, anchoring point
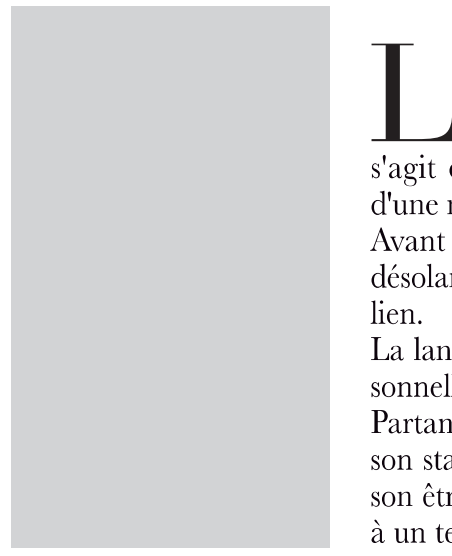

e lien entre le statut des langues et la démocratie n'est pas seulement - n'est pas essentiellement - de nature institutionnelle. Il est plus profond, plus fondamental, parce qu'il s'agit d'un lien moral et philosophique. En réalité deux faces d'une même médaille.

Avant d'illustrer ce lien par quelques indices et exemples aussi désolants que révélateurs, quelques mots sur la nature même du lien.

La langue est au cour de l'individu, à la fois son expression personnelle et sa voix - et sa voie de communication - vers l'Autre. Partant de là, la langue est pour chacun - bien davantage que son statut social, professionnel ou économique - indissociable de son être au monde, de sa personnalité. On peut certes s'identifier à un territoire, un État, une religion, une culture; mais ces identi- 
Langues et démocratie: un lien imprescriptible

G. DALGALIAN fications sont mouvantes et en perpétuel mouvement: cela fut toujours le cas et aujourd'hui c'est plus vrai que jamais.

En revanche, la langue reste un point d'ancrage que l'on peut rarement perdre, même si on peut lui rajouter des points d'ancrage supplémentaires, des secondes langues donc, tout au long de la vie. Il arrive aussi que l'enfant ait grandi avec plusieurs langues: ce fut mon cas. C'est bien plus fréquent à travers le monde qu'on ne croit: lorsque l'individu est bi- ou plurilingue, ses langues sont toutes constitutives de ses racines. Je préfère parler de «racines» plutôt que d'identité, mot qui renvoie à une vision trop essentialiste à mon goût.

«Racines» a d'abord le mérite d'être pluriel, toujours pluriel et toujours en évolution. 'Racines' a aussi le mérite de laisser chacun libre de pousser - suivant son propre tropisme - dans la direction qui lui convient sans le figer dans un moule identitaire.

Et c'est parce qu'elle exprime à la fois sa personnalité, sa façon d'être et sa liberté de choix que la langue est une facette indissociable de chacun, de sa dignité. Dignité, voilà un mot que d'aucuns trouveront ringard. Pourtant ne pas respecter cette dignité, exprimée dans la ou les langues de chacun, c'est en réalité refuser que l'Autre soit autre. Est pervers celui qui vit dans un monde sans Autre. Cette perversion conduit au déni de démocratie. La démocratie, c'est - bien avant les institutions - le respect. Lequel respect de l'autre s'exprime et se vérifie d'abord dans le respect de sa ou de ses langues.

Les situations de non-respect sont légion. Je vais en évoquer quelques-unes d'inégale gravité. Dans l'ordre de gravité croissante, je parlerai de l'ostracisme anti-accents régionaux, puis de l'effacement des langues régionales par des politiques scolaires éradicatrices, enfin du péril lié au nivellement linguistique à l'échelle planétaire.

L'ostracisme anti-accents régionaux naît d'une phobie. Un premier indice de cette phobie - un indice subtil, mais omniprésent se dévoile dans l'exclusion quasi systématique des accents régionaux chez les présentateurs et les chroniqueurs de nos médias. Exclusion que l'on n'observe ni sur les chaînes allemandes, suisses ou autrichiennes, ni ailleurs.

Pourtant on accepte et on apprécie dans nos médias les accents des étrangers qui parlent français, souvent assez correctement. Ce qui signifie que l'exclusion ne vise que les accents de France.

Ici il convient de noter que les locuteurs d'une langue régionale de France ne perdent pas tous leur accent, pourtant dévalorisé dès l'école. Les méridionaux occitanophones ou basques, par 
Langues et démocratie: un lien imprescriptible

G. DALGALIAN exemple, se font un plaisir de cultiver leur accent: ce n'est pas parce qu'ils seraient incapables de s'en débarrasser puisque beaucoup le font; non, c'est une forme de résistance culturelle que de marquer son occitanité, même lorsqu'on ne parle plus l'occitan.

D'ailleurs il n'y a pas un seul accent occitan, mais plusieurs selon les variantes dialectales. On retrouve ces différences dans le français des locuteurs du Midi: c'est toujours la transposition au français des traits prosodiques de l'occitan quelle que soit la variante locale.

Mais l'exclusion des accents n'est qu'un indice. Il y a plus grave. La phobie anti-langues (et celle anti-accents n'est que la partie émergée de l'iceberg) est la traduction d'un verrou idéologique plus profond que l'on peut formuler ainsi: le nivellement linguistique serait indispensable à l'unité de la nation française.

Nous voilà au cœur du blocage: on recherche l'unité et l'égalité dans l'uniformité totale des citoyens; on accorde au nivellement linguistique et culturel des attributs qu'il n'a pas. Le nivellement linguistique n'est pas synonyme de démocratie, ni de république: le nivellement linguistique est synonyme de non-respect du peuple dans ce qu'il a de plus profond, son parler. Or le respect est LE principe politique majeur; il est le fondement de toute démocratie.

Mais étendons-nous quelques instants sur les idées reçues qui ont fondé ou accompagné les politiques d'éradication des langues régionales. Elles reposent sur deux axiomes erronés.

Premier axiome erroné: le monolingue aurait une meilleure mâ̂trise du français et une seconde langue nuirait à ses compétences en langue nationale. Il m'appartient de combattre cette idée que malheureusement certains enseignants ont encore en tête. Car c'est le contraire qui est vrai. Pour plusieurs raisons.

Même lorsque l'enfant n'a pas bénéficié d'une transmission familiale et qu'il découvre cette langue dans une maternelle bilingue comme il en existe de plus en plus partout en France, ce n'est pas seulement une langue de plus à apprendre, (comme ce serait le cas au collège ou au lycée).

Il s'agit d'autre chose: une seconde langue précoce est une véritable formation cérébrale et intellectuelle. Pas seulement une ouverture et un complément de culture comme avec les langues tardives du collège et du lycée.

Pourquoi parler de formation cérébrale? Parce qu'à cet âge précoce on n'acquiert pas la langue de façon consciente et volontaire, mais dans le vécu des jeux et des interactions, des comptines et des chansons. De telle façon que l'enfant pratique des allers- 
Langues et démocratie: un lien imprescriptible

G. DALGaLIAN retours permanents entre ses deux langues inconsciemment et efficacement, sans traduire, ni réfléchir, mais dans une libre reformulation de ce qu'il ressent ou veut exprimer. Avec ses affabulations et ses omissions volontaires.

Ces allers-retours permanents l'amènent à saisir que derrière des formes différentes s'expriment des contenus identiques ou similaires. Ce, malgré quelques différences culturelles. Donc l'élève d'une classe bilingue a très tôt touché du doigt la relativité des formes et des mots et l'universalité très large des contenus et des sentiments. Voilà une formation de l'esprit qu'aucune acquisition tardive ne garantit avec autant de profondeur et d'efficacité qu'une éducation bilingue précoce.

Ainsi tout petit déjà, j'ai appris dans un vécu que l'Autre n'est qu'une variante humaine comme moi. Et qu'il s'exprime comme moi avec ses différences.

L'avantage que donne la maîtrise d'une seconde langue, Johann Wolfgang Goethe l'avait déjà énoncée en son temps: "On ne connaît bien sa langue que lorsqu'on en parle d'autres".

Pendant très longtemps les officiels de l'Éducation nationale des $19 \mathrm{e}$ et 20e siècles ont avancé l'argument selon lequel "les dialectes ne sont pas de vraies langues, parce qu'elles n'ont pas de grammaire" (sic). Ceci n'est rien d'autre qu'une ineptie. En effet la grammaire n'est pas cette description de la langue que prodigue l'école primaire.

La grammaire arrive dans un cerveau d'enfant à l'oral et avant l'école, dès qu'il observe, engrange et reproduit les féminins, les pluriels, les formes des verbes, les constructions de phrases et l'ordre des mots, les prépositions ou ailleurs les déclinaisons.

C'est toute la morphosyntaxe que l'enfant a intériorisée et automatisée entre zéro et sept ans et c'est justement cette dimension de la langue qui deviendra plus difficile à acquérir (les bilinguismes tardifs existent aussi, mais ils n'ont pas les mêmes effets sur la formation, notamment cérébrale des individus). D'où aussi la plus grande fluidité des langues précoces, lorsque les automatismes ont été construits dans un âge tendre, l'âge du langage.

Bref, une langue sans grammaire, ça n'existe pas.

Or la construction d'automatismes linguistiques dans une langue maternelle - ou dans deux langues - est ensuite transférable à d'autres langues: ce sont précisément les automatismes de la tendre enfance qui permettront la construction de nouveaux automatismes pour les langues tardives du collège et du lycée. Ge qui fait du bilingue précoce un plurilingue en herbe, parce que disposant d'un double stock de sons, de mots et de structures - ses 
Langues et démocratie: un lien imprescriptible

G. DALGALIAN transferts à d'autres langues seront plus aisés et plus rapides que chez le monolingue. De même on n'est pas meilleur francophone parce qu'on est monolingue. C'est le contraire.

Second axiome erroné: le locuteur monolingue serait un meilleur citoyen que les autres. Cela n'est jamais dit explicitement, mais c'est toujours sous-jacent. Or cela fait bon marché de tous nos auteurs, artistes, savants, chanteurs et comédiens - dont les noms peuplent nos écrans et notre histoire - qui ont enrichi la création française grâce à leur bilinguisme et biculturalisme originel.

Sans parler de tous ces 'morts pour la France' qui étaient locuteurs d'une langue africaine ou autre et pour qui le français était leur seconde langue.

Ces deux axiomes sont des constructions. Elles on été élaborées au fil du temps et des méandres et palinodies de l'Histoire. Elles n'ont aucune base en sciences humaines et sociales. Pourtant si elles ont trop longtemps intoxiqué la pensée - ou la non-pensée c'est que derrière les idées reçues se cachent les phobies tenaces que j'ai évoquées.

Mon plaidoyer en faveur des langues régionales vaut également pour les langues d'origine des enfants des immigrations anciennes ou récentes, du moins lorsque la demande est réelle; ceci pourtant ne fait sens que si ces élèves en désir de bilinguisme ne sont pas enfermés dans un ghetto ethnique ou linguistique, ce qui fut le cas avec les ELCO (langues et cultures d'origine) où les petits Portugais ou les petits arabophones se retrouvaient entre eux.. L'accès aux langues d'origine, comme pour les langues régionales, ne doit exclure personne et s'ouvrir à tous.

Dans ce panorama trop rapide des politiques linguistiques et scolaires, je m'en voudrais d'oublier le cas le plus désolant de tous: le statut des parlers d'Alsace et de Moselle dont la référence écrite est l'allemand.

Pourquoi désolant? D'abord parce que ces langues - dont l'allemand - sont insuffisamment promus dans l'institution scolaire et universitaire de leur propre région.

Insuffisamment par rapport aux nombreuses relations de l'Alsace et de la Moselle avec leurs voisins allemands et suisses; insuffisamment au vu du grand nombre d'emplois transfrontaliers perdus, soit par manque de bilingues, soit parce que les bilingues alsaciens ou franciques n'ont plus la maitrise du standard, l'allemand.

À ce point précis je souhaite montrer pourquoi ce serait une hérésie de vouloir opposer l'allemand à l'alsacien: ce serait se tirer une balle dans le pied, car ici comme dans toutes les régions germanophones et dialectophones l'allemand est le fond de réserve et le 
Langues et démocratie: un lien imprescriptible

G. Dalgalian prolongement naturel des dialectes locaux.

Même en Suisse les journaux sont de langue allemande standard; d'ailleurs ils ne disent pas Hochdeutsch mais Schrifdütsch, allemand écrit.

Les Dernières Nouvelles d'Alsace et l'autre quotidien, l'Alsace, ont longtemps eu une édition bilingue faisant une large place à l'allemand standard. Hélas les politiques linguistiques combinées à la disparition des vieux locuteurs ont abouti à cette régression: la presse quotidienne bilingue n'existe plus; elle est remplacée par un supplément hebdomadaire envoyé aux seuls abonnés.

Ici un rappel historique indispensable: pendant très longtemps ni le côté officiel français, ni les locuteurs alsaciens n'ont fait de distinction entre le parler oral et le standard allemand. Jusqu'à la fin du 19e siècle, c'est le terme Ditsch ou Deutsch qui est utilisé pour désigner aussi bien les formes parlées que les formes écrites en standard allemand.

Paradoxalement, c'est sous le 2e Reich après 1870 que, l'Alsace n'ayant pas le même statut politique que les autres régions de l'Empire, apparaît l'expression 'Elsässerdeutsch' et plus tard 'Elsässisch'. Et c'est bien plus tard, après 1918, que la politique linguistique française va s'engouffrer dans ce distinguo pour mieux estomper le lien organique entre l'oral alsacien et l'écrit allemand. Mais quand donc a eu lieu cette entrée de la langue allemande dans l'univers français? Cela remonte à 1648, date du premier rattachement de l'Alsace-Moselle au royaume de France en application du Traité de paix de Westphalie. On peut largement attribuer à la diplomatie et à l'intervention militaire de Richelieu d'abord, de Mazarin ensuite, l'entrée de la langue allemande dans le royaume de France. Et pour plusieurs siècles sans interruption. L'exclusion de cette langue est donc relativement récente et n'a d'équivalent que la 'défrancisation' - la 'Entweelschung' - pratiquée par les nazis entre 1940 et 1945.

Revenons-en à la politique scolaire officielle d'aujourd'hui. Elle vient encore aggraver les effets de l'éradication tant des dialectes que de l'allemand: le nombre de vraies filières bilingues précoces français/allemand - bien qu'en légère progression en Alsace depuis peu - ne correspond ni aux potentialités en nombre de locuteurs, ni aux besoins économiques, commerciaux et professionnels de l'Alsace. En Moselle la situation est pire encore. Que l'on compare cela au Land de Sarre où le français est enseigné dès l'école primaire.

Oui, l'allemand est une langue de France dans sa double face, dialectale et standard; ce depuis plus de trois siècles. Certes la pulsion 
Langues et démocratie: un lien imprescriptible

G. DALGalian de nivellement s'est appuyée sur un lourd passé de guerres francoallemandes. Mais n'est-il pas venu le temps de faire la distinction entre langue et histoire, entre le passé et le présent? Il est temps de redonner à la langue allemande sa place de choix non seulement comme langue du voisin et ami, mais aussi comme langue écrite et arrière-plan culturel des locuteurs de l'alsacien et du mosellan, lorsqu'ils le désirent.

J'en arrive à cette menace de loin la plus grave: la vertigineuse perte des langues au cours des temps modernes et le nivellement linguistique et culturel observé partout, notamment avec l'omniprésence de l'anglais dominant.

Pourtant la tendance au nivellement et à l'uniformisation n'a pas commencé seulement avec l'anglais. Elle a des antécédents sur la planète entière et a aussi une riche histoire dans l'ère française et francophone à travers le temps et l'espace.

On peut s'interroger sur le paradoxe de notre pays qui possède le plus grand gisement de langues de toute l'Europe de l'Ouest et qui le néglige. Seul le mépris a pu conduire à être indifférent à une telle richesse, tantôt en les ignorant, tantôt en les rabaissant à des tares. Ce qui fut le cas, de l'Abbé Grégoire à Jules Ferry.

Cependant il est inutile de taire la responsabilité des opinions publiques et notamment des parents d'élèves qui ont leur part dans l'alignement sur ce qui leur apparait comme le plus fort capital culturel et social, désormais presque toujours au bénéfice de l'anglais. Ge capital social semble décider de plus en plus souvent ce qui fait qu'une langue est légitime ou non.

C'est une conception exagérément utilitariste qui conduit tant de parents partout à choisir l'anglais en premier, même en filières précoces ou semi-précoces.

Outre que ce choix tient rarement compte de l'environnement linguistique réel, il s'apparente à un (mauvais) pari sur l'avenir de l'enfant. Il procède de la représentation erronée qui voit en chaque progéniture un futur ingénieur, manager ou informaticien, sans se douter que le moment venu cet enfant sera peut-être musicien, enseignant ou garagiste (et que pour tous il serait plus bénéfique de démarrer dans la vie avec les langues de l'environnement réel).

Non seulement cette vision - utilitaire jusqu'au conformisme tourne le dos à la liberté de l'enfant, à ses libres choix et talents, mais en outre elle occulte cette donnée: le bilingue précoce apprendra plus vite et mieux l'anglais en 3e position que son camarade monolingue qui aborde l'anglais en lère langue vivante au collège. 
Langues et démocratie: un lien imprescriptible

G. DALGaLIAN
Avec ce résultat qu'aucune langue ne peut faire aujourd'hui obstacle à l'anglais, tandis que l'anglais nourrit chez beaucoup l'illusion de suffire en toute situation. Avec pour conséquence que trop souvent l'anglais devient de facto un obstacle aux autres langues. Notamment par les choix de certains parents.

Bref, la clé anglaise n'est pas la clé universelle. La seule clé universelle, c'est le plurilinguisme dont l'anglais sera une composante.

Mais la critique d'une uniformisation par un anglais dominant ne saurait épuiser un problème plus profond et plus complexe. Je veux parler de la régression généralisée de la diversité linguistique et culturelle. C'est bien d'un recul de l'humain dans ce qui est l'essentiel.

Or il semble que l'on soit passé très largement à côté de l'essentiel: la diversité linguistique et culturelle est un prolongement de la biodiversité.

Voyons comment cela se comprend: au cours des 300000 dernières années Homo a ajouté à son patrimoine biologique initial tout un nouveau patrimoine d'innovations et d'apprentissages, notamment culturels et linguistiques, transmis de génération en génération et renouvelé et enrichi par les nouvelles générations. Certains auteurs ont appelé ces avancées une 'biodiversité culturelle'.

Mais ce terme n'est pas satisfaisant: il montre bien la filiation entre la biodiversité et la diversité culturelle, mais il gomme la différence de nature; il m'a paru plus judicieux de forger un terme spécifique qui marque cette spécificité du culturel et du linguistique. J'ai donc proposé le terme de 'Glossodiversité'.

Quoi qu'il en soit, la glossodiversité est bien le prolongement de la biodiversité, mais sous des formes inédites: l'évolution darwinienne ne pouvait pas s'arrêter au moment même où une espèce, la nôtre, avait mis en œuvre des capacités nouvelles d'évolution et d'auto-transformation. Avec leurs langues, leurs cultures et leurs techniques, les humains avaient changé le rapport de l'homme à son environnement.

À la réflexion, il eût été impensable que, toutes les espèces ayant évolué et s'étant diversifiées depuis des millions d'années sous pression constante de l'environnement, cette diversification s'arrête d'un coup avec l'émergence d'Erectus et de Sapiens. C'est forcément le contraire qui devait se produire: doté d'un nouveau cerveau et de nouveaux savoir-faire, Homo ne pouvait pas ne pas modifier son environnement et se modifier soi-même dans une interaction permanente tous azimuts.

Cela devint une vertigineuse accélération dans l'évolution et la 
Langues et démocratie: un lien imprescriptible

G. DALGALIAN diversification des humains et, partant, de leur environnement. Pour le meilleur et pour le pire!

Pour le pire aussi, car ces capacités nouvelles peuvent ouvrir sur de vrais progrès, mais aussi sur des régressions, notamment en raison désormais de l'énorme pression de la globalisation. L'une de ces régressions concerne justement les langues et les cultures. C'est-à-dire l'essentiel de la psyché humaine!

La glossodiversité n'est pas un 'plus' dont on pourrait se passer au profit d'un nivellement, d'une uniformisation linguistique devant soi-disant permettre une meilleure communication planétaire. Ce qui se ferait au détriment des langues dites minoritaires, puis de langues nationales, y compris de grandes langues.

Cette uniformisation planétaire fait que le combat pour les langues est indivisible: c'est l'avenir de toutes les langues qui est en jeu.

Or la glossodiversité a été et reste inséparable de toute l'hominisation et il n'y a pas d'humanité sans cette diversité intrinsèque. Dès l'aube de l'humanité, l'hominisation a été synonyme de diversification et cela s'est multiplié avec le temps.

Le foisonnement des profils est aussi source de diversité psychologique et intellectuelle et c'est ce foisonnement intellectuel qui constitue le fondement de toute démocratie.

En termes actuels, il importe de prendre la mesure de la force et de la légitimité de cette diversité. En clair, aucune langue ne peut prétendre être la clé universelle. La seule clé universelle, c'est le plurilinguisme. Plurilinguisme des individus et des sociétés! 